EPJ Web of Conferences 60, 13013 (2013)

DOI: $10.1051 /$ epjconf $/ 20136013013$

(C) Owned by the authors, published by EDP Sciences, 2013

\title{
Higher moments of net kaon multiplicity distributions at RHIC energies for the search of QCD Critical Point at STAR
}

\author{
Amal Sarkar ${ }^{1, a}$ (for the STAR collaboration) \\ ${ }^{1}$ Indian Institute of Technology Bombay \\ Mumbai, India - 400076
}

\begin{abstract}
In this paper we report the measurements of the various moments mean $(M)$, standard deviation $(\sigma)$, skewness $(\mathrm{S})$ and kurtosis $(\kappa)$ of the net-Kaon multiplicity distribution at midrapidity from $\mathrm{Au}+\mathrm{Au}$ collisions at $\sqrt{s_{N N}}=7.7$ to $200 \mathrm{GeV}$ in the STAR experiment at RHIC in an effort to locate the critical point in the QCD phase diagram. These moments and their products are related to the thermodynamic susceptibilities of conserved quantities such as net baryon number, net charge, and net strangeness as also to the correlation length of the system. A non-monotonic behavior of these variable indicate the presence of the critical point. In this work we also present the moments products $\mathrm{S} \sigma, \kappa \sigma^{2}$ of net-Kaon multiplicity distribution as a function of collision centrality and energies. The energy and the centrality dependence of higher moments of net-Kaons and their products have been compared with it's Poisson expectation and with simulations from AMPT which does not include the critical point. From the measurement at all seven available beam energies, we find no evidence for a critical point in the QCD phase diagram for $\sqrt{s_{N N}}$ below $200 \mathrm{GeV}$.
\end{abstract}

\section{Introduction}

One of the fundamental goals of the heavy-ion collision experiment is to map the QCD phase diagram as a function of temperature and baryon-chemical potential $\left(\mu_{B}\right)[1]$. Many QCD models based, admit the existence of a critical point which is the quest of present investigations at the Relativistic Heavy Ion Collider (RHIC) at BNL. Finite temperature lattice QCD calculations at baryon chemical potential $\mu_{B}=0$ suggest a crossover above a critical temperature from a system with hadronic degrees of freedom to a system where the relevant degrees of freedom are quarks and gluons[2,3]. Several QCD based calculations find the quark-hadron phase transition to be first order at large $\mu_{B}$. The point in the QCD phase plane ( $T$ vs $\mu_{B}$ ) where the first order phase transition ends is the QCD critical point (CP) [4]. At the critical point the first order transition becomes continuous, resulting in long range correlation and fluctuation at all length scale. Such properties of state open possibilities for distinct experimental signatures which can be used to discover the critical point. Current theoretical calculations are highly uncertain about location of the CP. Lattice QCD calculations at finite $\mu_{B}$ face numerical challenges in computing. The experimental plan is to vary the center of mass energy $\left(\sqrt{s_{N N}}\right)$ of heavy-ion collisions to scan the phase plane and at each energy, search for signatures of the critical point that could survive the time evolution of the system.

The correlation length $(\xi)$ and the magnitude of the fluctuations diverge at the critical point $[5,6]$. However,

\footnotetext{
a e-mail: amal@rcf.rhic.bnl.gov
}

in heavy-ion collision finite size and time effect brings down the value of $\xi$ in the range of $2-3 \mathrm{fm}$ from infinity [7]. It has been shown that the higher non Gaussian moments (as they depend on higher powers of $\xi$ ) of fluctuations of conserved quantities are much more sensitive to correlation length than variances $\left(\sigma^{2}=(\Delta N)^{2} ; \Delta N=\right.$ $N-M ; M$ is the mean) (which vary only as $\xi^{2}$ ) [9-11]. For example, the skewness $\left.\left(\mathrm{S}=\left\langle(\Delta N)^{3}\right\rangle / \sigma^{3}\right)\right)$ and kurtosis $\left(\kappa=\left[\left\langle(\Delta N)^{4}\right\rangle / \sigma^{4}\right]-3\right)$ have been shown to be related to the $\xi^{4.5}$ and $\xi^{7}$, respectively [9-11]. A crossing of the phase boundary can manifest itself by a change of sign of $\mathrm{S}$ as a function of energy density[10]. The moments of the net distribution contains a system volume dependence. To cancel out these volume dependency we construct moment products like $\mathrm{S} \sigma$ and $\kappa \sigma^{2}$. These moment product can also write as ration of cumulants, $\mathrm{S} \sigma=\frac{C_{3}}{C_{2}}$ and $\kappa \sigma^{2}=\frac{C_{4}}{C_{2}}[11]$. These volume independent moment product having sensitivity to the correlation length $(\xi)$ as $\mathrm{S} \sigma \propto \xi^{2.5}$ and $\kappa \sigma^{2} \propto \xi^{5}[11]$. Close to the critical point, models predict the net-baryon, net-strangeness and netcharge number distributions to be non-Gaussian and susceptibilities to diverge causing $\mathrm{S} \sigma$ and $\kappa \sigma^{2}$ to deviate from being constants and have large values.

Lattice calculations and QCD-based models show that moments of net-conserved (baryons(B), strangeness(S) and charge $(\mathrm{S}))$ distributions are related to it's conserved number susceptibilities $\left(\chi_{x}=\frac{\left\langle\left(\Delta N_{x}\right)^{2}\right\rangle}{V T}\right.$; where, $\mathrm{x}$ represents $\mathrm{B}, \mathrm{S}$ and $\mathrm{Q}, \mathrm{V}$ is the volume) [3,12]. Volume independent moment product $\mathrm{S} \sigma$ and $\kappa \sigma^{2}$ are the ration of third $\left(\chi_{x}^{(3)}\right)$ to second $\chi_{x}^{(2)}$ order and forth $\chi_{x}^{(4)}$ to second $\chi_{x}^{(2)}$ order suscep- 
tibilities[3,12]. Experimentally measuring event-by-event all produced conserved quantities is very difficult. However, proton, kaons and charge are measurable for their proxy of the conserved quantities. Event-by-event the netKaon multiplicity ( $\Delta N_{K}=N_{K^{+}}-N_{K^{-}}$) distribution is measurable for the proxy of the net-strangeness. Theoretical calculations have shown that $\Delta N_{K}$ fluctuations reflect the singularity of the charge and Strangeness number susceptibility as expected at the critical point $[12,13]$.

In this work, we report the first measurement of the moments of the net-Kaon multiplicity distributions as a function of baryon chemical potential which was varied from 410 to $20 \mathrm{MeV}$ by changing the $\sqrt{s_{N N}}=7.7,11.5$, 19.6, 27, 39, 62.4 and $200 \mathrm{GeV}$ in $\mathrm{Au}+\mathrm{Au}$ collisions [14]. The data were taken by the STAR experiment in 2010 and 2011 as a part of the beam energy scan (BES) program at RHIC [14]. Different combination of these moment product such as $\mathrm{S} \sigma$ and $\kappa \sigma^{2}$ have been calculated (to cancel out the dependence on the volume) as collision centrality[5,9]. QCD model calculations with critical point expect a non-monotonic dependence of these moments products near the critical point [9].

\section{Analysis Details}

The STAR(Solenoidal Tracker At RHIC) experiment at Brookhaven National Laboratory provides excellent particle identification and large uniform acceptance at $\sqrt{s_{N N}}=7.7-200 \mathrm{GeV}$ [14-16]. Ionization energy loss $d E / d x$ of charged particles in the Time Projection Chamber (TPC) was used to identify charged kaons by comparing it to the theoretical (parameterized) expectation along with a $\mathrm{m}^{2}$ cut from the information of Time-of-Flight (TOF) detector $[15,16]$. The analysis was carried out with the event-by-event identified positively charged kaons $\left(K^{+}\right)$and negatively charged kaons $\left(K^{-}\right)$in full azimuthal coverage for collisions occurring within $30 \mathrm{~cm}$ of the TPC center along the beam line. The $\Delta N_{K}$ measurements are carried out within the pseudo-rapidity range $|\eta|<0.5$ in the range $0.2<p<1.6 \mathrm{GeV} / \mathrm{c}$. To reduce the contamination from secondary kaons, we required each $K^{+}\left(K^{-}\right)$track to have a minimum $p_{T}$ of $0.2 \mathrm{GeV} / \mathrm{c}$ and a distance of closest approach (DCA) to the primary vertex of less than $1 \mathrm{~cm}$. To suppress the contamination from other particles we use $\left|n \sigma_{K}\right|<2.0$ where,

$$
n \sigma_{x}=\frac{1}{R} \log \frac{\left.\langle d E / d x\rangle\right|_{\text {measured }}}{\left.\langle d E / d x\rangle\right|_{\text {expected }}}
$$

For centrality selection we have used uncorrected charged particle multiplicity within $0.5<|\eta|<1.0$, to avoid the autocorrelation. For each centrality, the average numbers of participants $\left(\left\langle N_{\text {part }}\right\rangle\right)$ are obtained by Glauber model calculations. The results are corrected for the finite centrality bin width effects[17]. The numbers of events analyzed are in the table below.
Table 1. The numbers of events analyzed for $\mathrm{Au}+\mathrm{Au}$ collisions at $\sqrt{s_{N N}}=7.7$ to $200 \mathrm{GeV}$ in the STAR experiment at RHIC

\begin{tabular}{lll}
\hline $\begin{array}{l}\text { Energy } \\
\text { (in GeV) }\end{array}$ & $\begin{array}{l}\text { Number of Events } \\
\text { (in Million) }\end{array}$ & Year of \\
\hline 7.7 & 2.4 & 2010 \\
11.5 & 7.5 & 2010 \\
19.6 & 17 & 2011 \\
27 & 34 & 2011 \\
39 & 40 & 2010 \\
62.4 & 44 & 2010 \\
200 & 200 & 2010 \\
\hline
\end{tabular}

\section{Results}

The raw net-Kaon $\left(\Delta N_{K}=N_{K^{+}}-N_{K^{-}}\right)$multiplicity distribution for various collision centralities from 70-80\%, 30$40 \%$, and $0-5 \%$ in $\mathrm{Au}+\mathrm{Au}$ collisions at $\sqrt{s_{N N}}=7.7-200$ $\mathrm{GeV}$ at midrapidity $(|\eta|<0.5)$, are shown in the Figure 1 . $\Delta N_{K}$ was not corrected for reconstruction efficiency. The

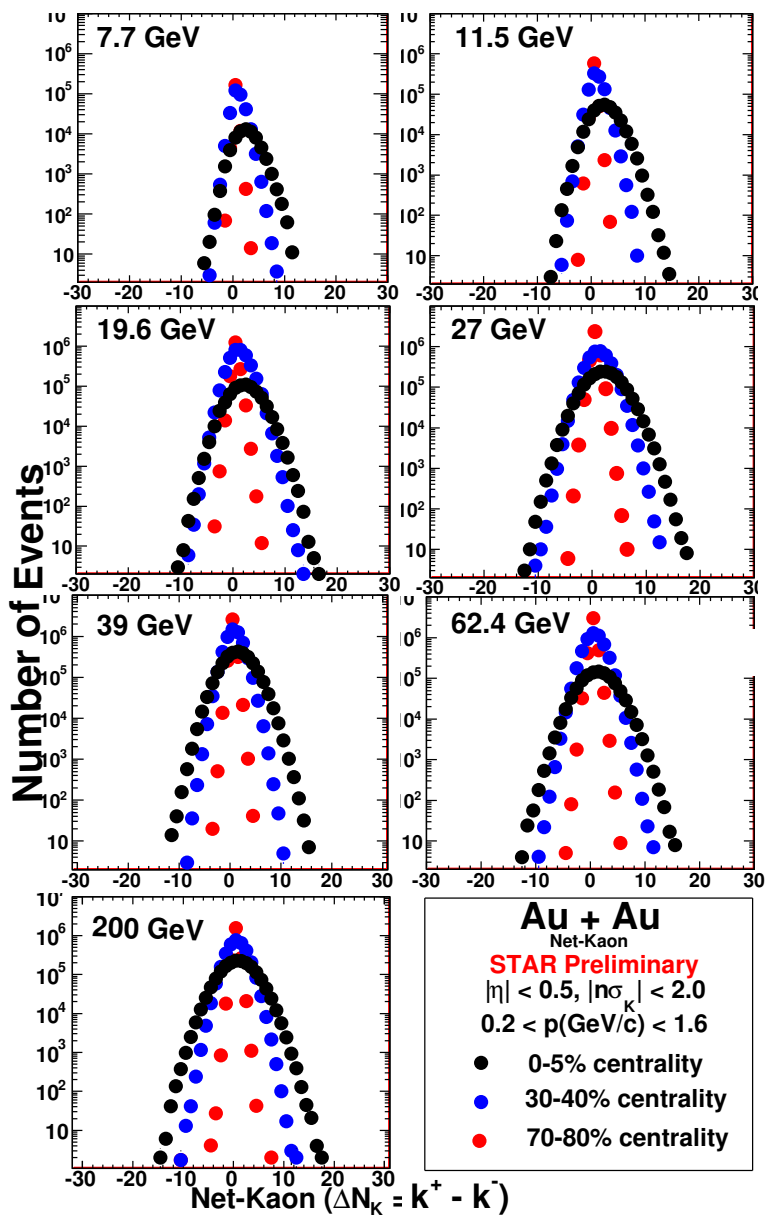

Figure 1. $\Delta N_{K}$ multiplicity distribution in $\mathrm{Au}+\mathrm{Au}$ collisions at $\sqrt{s_{N N}}=7.7-200 \mathrm{GeV}$ for various collision centralities at midrapidity $(|\eta|<0.5)$ and the moments calculated from these distribution after applying the bin-width correction[17]

mean of net-Kaon $\left(\Delta N_{K}\right)$ distribution shifts towards zero from low to high energies. 
The four moments ( $M, \sigma, S$, and $\kappa$ ) of the $\Delta N_{K}$ distribu-
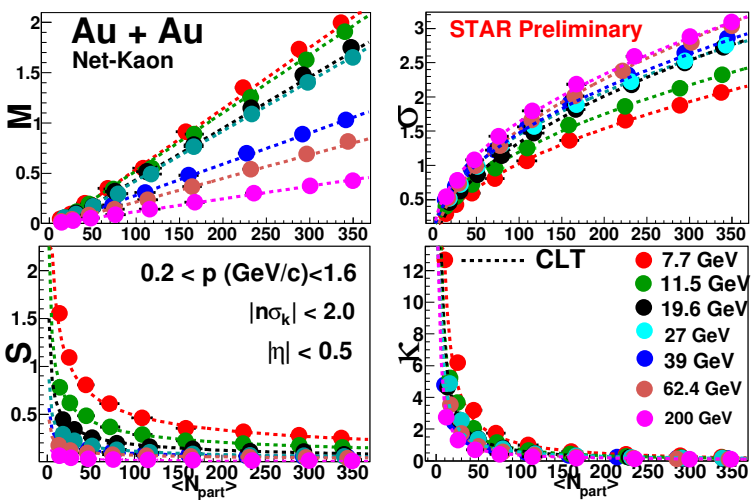

Figure 2. Centrality dependence of moments of $\Delta N_{k}$ distributions for $\mathrm{Au}+\mathrm{Au}$ collisions at $\sqrt{s_{N N}}=7.7-200 \mathrm{GeV}$. The doted lines are the expected values from the central limit theorem. Error bars are statistical only from Delta Theorem[18].

tions at various collision energies are plotted as a function of average number of participants $\left\langle N_{\text {part }}\right\rangle$ in Figure 2.The moments are calculated from the $\Delta N_{K}$ distributions after correcting for the finite centrality bin width effects[17]. The $\mathrm{M}$ shows a linear variation with $\left\langle N_{\text {part }}\right\rangle$ and increases as $\sqrt{s_{N N}}$ decreases. The $\sigma$ increases with $\left\langle N_{\text {part }}\right\rangle$. The values are increasing with the increasing beam energies. The $\mathrm{S}$ is positive and decreases as $\left\langle N_{\text {part }}\right\rangle$ increases for a given collision energy. The values also decrease as $\sqrt{s_{N N}}$ increases. This indicates that the distributions become symmetric for more central collision and for higher beam energies. The $\kappa$ decreases as $\left\langle N_{\text {part }}\right\rangle$ increases, and also decrease as $\sqrt{s_{N N}}$ increase in all beam energies studied. The fitted doted lines are the expected values from the central limit theorem (CLT), which varies as volume's $x, \sqrt{x}, \frac{1}{\sqrt{x}}$ and $\frac{1}{x}$ respectively. Error bars are statistical only, calculated from Delta theorem [18].

The centrality dependence $\mathrm{S} \sigma$ and $\kappa \sigma^{2}$ are shown in Fig-

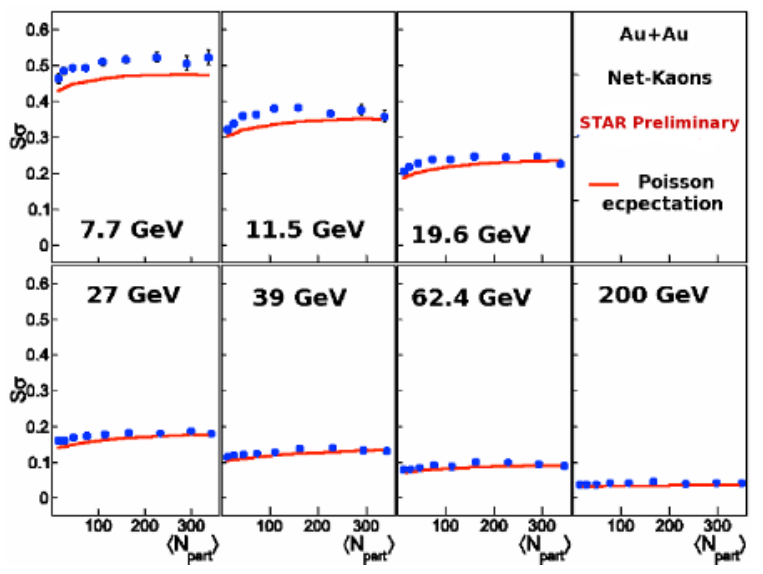

Figure 3. Centrality dependence of $\mathrm{S} \sigma$ of $\Delta N_{k}$ in Au+Au collisions at $\sqrt{s_{N N}}=7.7-200 \mathrm{GeV}$ compared to its Poisson expectation.

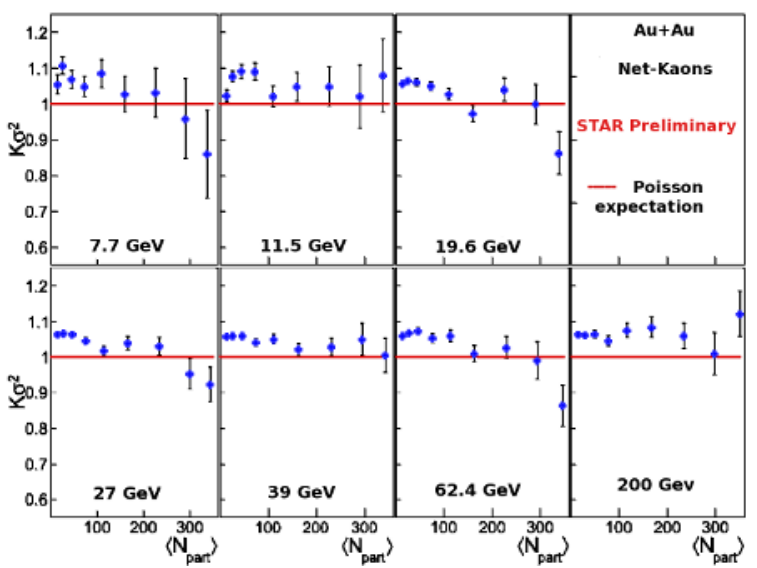

Figure 4. Centrality dependence of $\kappa \sigma^{2}$ for $\Delta N_{k}$ in Au+Au collisions at $\sqrt{s_{N N}}=7.7-200 \mathrm{GeV}$ compared to its Poisson expectation.

ure 3 and Figure 4 compared with the baseline from Poisson statistics for the seven available beam energies. The Poisson baseline has been calculated from the mean value of the $N_{K^{+}}$and $N_{K^{-}}$distribution. There is a very small increasing centrality dependency in $\mathrm{S} \sigma$ value towards most central collision which is within $15 \%$. S $\sigma$ value is greater than Poisson baseline for beam energy below $200 \mathrm{GeV}$. S $\sigma$ increase with decreasing collision energies. Volume independent product $\kappa \sigma^{2}$ is independent of centrality with in $10 \%$. Within the statistical uncertainty $\kappa \sigma^{2}$ value matches with the Poisson expectation value in all centrality for all available beam energies.

In Figure 5 the energy dependency of the volume inde-

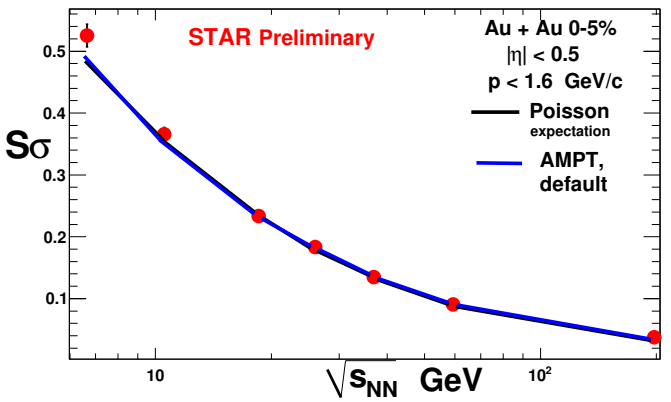

Figure 5. $\sqrt{s_{N N}}$ dependence of $\mathrm{S} \sigma$ for net-Kaon distributions measured at RHIC for the top central $0-5 \% \mathrm{Au}+\mathrm{Au}$ collisions for available energies. The results are compared to AMPT non$\mathrm{CP}$ model calculation and with the Poisson expectation.

pendent moment products $\mathrm{S} \sigma$ for the top central $0-5 \%$ $\mathrm{Au}+\mathrm{Au}$ collisions are shown. The data has been compared with the Poisson baseline and with the AMPT model result. $\mathrm{S} \sigma$ value matches with Poisson expectation and AMPT value for the top $0-5 \%$ central collision.

The volume independent product $k \sigma^{2}$ for the top central $0-5 \% \mathrm{Au}+\mathrm{Au}$ collisions for available energies are plotted in the Figure.6. Within the statistical uncertainty, $\kappa \sigma^{2}$ 


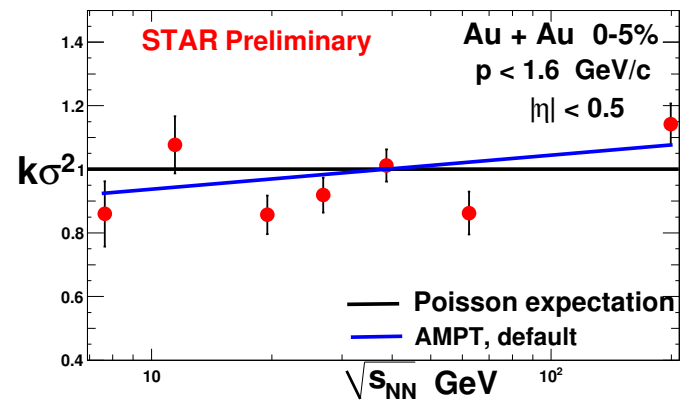

Figure 6. $\sqrt{s_{N N}}$ dependence of $\kappa \sigma^{2}$ for net-Kaon distributions measured at RHIC for the top central $0-5 \% \mathrm{Au}+\mathrm{Au}$ collisions for available energies. The results are compared to AMPT non$\mathrm{CP}$ model calculation and with the Poisson expectation.

value matches with AMPT default value for the top $0-5 \%$ central collision. $\kappa \sigma^{2}$ value matches with Poisson baseline for the top $0-5 \%$ central collision.

\section{Summary}

The first measurements of the higher moments kurtosis $(\kappa)$, skewness $(\mathrm{S})$, and variance $\left(\sigma^{2}\right)$ of net-Kaon multiplicity $\left(\Delta N_{K}=N_{K^{+}}-N_{K^{-}}\right)$distributions at mid-rapidity $(|\eta|<0.5)$ in $0.2<p<1.6 \mathrm{GeV} / \mathrm{c}$ for $\mathrm{Au}+\mathrm{Au}$ collisions at $\sqrt{s_{N N}}=7.7,11.5,19.6,62.4$, and $200 \mathrm{GeV}$ corresponding to baryon chemical potentials $\left(\mu_{B}\right)$ between 400 and 20 $\mathrm{MeV}$ were shown. The centrality dependence of moments follows the central limit theorem well. New observables $\mathrm{S} \sigma$ and $\kappa \sigma^{2}$ derived from the $\Delta N_{K}$ distribution to search for the critical point in heavy-ion collisions are discussed. $\mathrm{S} \sigma$ values are found to be independent of centrality as a function of $\left\langle N_{\text {part }}\right\rangle$ for all available collision energies within $15 \%$. $\kappa \sigma^{2}$ values are also found to be constant within the statistical uncertainties as a function of $\left\langle N_{\text {part }}\right\rangle$ for all collision energies studied. $\mathrm{S} \sigma$ and $\kappa \sigma^{2}$ are consistent with expectations from AMPT model without the critical point in top central $0-5 \% \mathrm{Au}+\mathrm{Au}$ collisions. No significant enhancement of moment products was observed compared to the Poisson baseline at presently available energies.

\section{References}

[1] J. Adams et al., Nucl. Phys. A757, 102 (2005).

[2] Y. Aoki, et al., arXiv:1007.2613 (2010).

[3] M. Cheng, et al., Phys. Rev. D 79 (2009) 074505

[4] M.A. Stephanov, Prog. Theor. Phys. Suppl. 153, 139 (2004).

[5] M. M. Aggarwal, et al., arXiv:1007.2613 (2010).

[6] M. A. Stephanov, Phys. Rev. Lett. 107, 052301(2011).

[7] B. Berdnikov et al., Phys. Rev. D 61, 105017 (2000).

[8] STAR Collaboration, Phys. Rev. Lett 105, 022302 (2010).

[9] M. A. Stephanov, Phys. Rev. Lett. 102, 032301 (2009).

[10] C. Athanasiou, et al, Phys. Rev. D 82, 074008 (2010).

[11] S. Gupta, arXiv:0909.4630 [nucl-ex] (2009).

[12] F. Karsch et al., Phys. Lett. B 695, pp 136-142 (2011).

[13] Y. Hatta and M. A. Stephanov, Phys. Rev. Lett. 91, 102003 (2003).

[14] STAR Collaboration, B. I. Abelev et al., Phys. Rev. C 81, 024911 (2010).

[15] STAR Collaboration, Nucl.Instrum.Meth. A558, pp. 419-429 (2006).

[16] STAR Collaboration, Nuclear Physics A, (vol. 774, pp. 956-958,2006).

[17] X. Luo [STAR Collaboration], J. Phys. Conf. Ser. 316, 445012003 (2011).

[18] X. Luo, J. Phys. G 39, 025008 (2012)[arXiv: 1109.0593]. 